\title{
Preventive Health Application to Increase Breastfeeding
}

\author{
Jimi Francis, PhD, IBCLC, RDN ${ }^{1}$ and Darby Dickton, DO, CLC ${ }^{2}$
}

\begin{abstract}
Background: Mother-infant dyads are not meeting recommended breastfeeding goals. There is lack of consensus regarding any program's ability to increase breastfeeding duration to meet the recommended guidelines, and effective strategies are needed moving forward to help families meet those goals. Primary prevention utilizing consistent visits with lactation professionals with a group of new mothers may efficiently address this care gap.

Methods: Mother-infant dyads attending a newborn care clinic for 6 weeks received feeding assessment and standard-of-care guidance from an Internationally Board-Certified Lactation Consultant (IBCLC). Regression analysis was completed with outcome variables "exclusive direct breastfeeding" (EDB) and "any breastfeeding" (AB) at 6 weeks postpartum (PP) and odds ratios were calculated.

Results: The number and timing of IBCLC visits was correlated with EDB at 6 weeks PP. Maternal/infant dyads seen at day 3 had 2.5 times higher odds of EDB at 6 weeks than those not receiving IBCLC standard-ofcare feeding assessment. Dyads seen at days 3 and 14 had 3.4 times higher odds of EDB than those with less follow-up. Bottle use correlated with decreased odds of AB at 6 weeks PP; similarly, dyads seen only at day 14 PP had decreased odds of EDB. When looking at timing of the first involvement, dyads seen at 3 days had higher odds of EDB than dyads first seen at day 14 PP.

Conclusions: This primary prevention strategy of early minimal intervention using an IBCLC increased the odds of EDB and $\mathrm{AB}$ at 6 weeks PP. These data support the conclusion that early feeding assessment the first 2 weeks PP with an IBCLC may increase breastfeeding at 6 weeks PP.
\end{abstract}

Keywords: breastfeeding outcomes; early assessment, preventive health care, lactation consultant

\section{Introduction}

$\mathbf{H}$ ISTORICALLY, BREASTFEEDING FOR mammalian species meant survival, and is acknowledged as the best source of nutrients and immune support for most infants. And although there are policies and practices in many localities that encourage and promote breastfeeding, many mother-infant dyads are not meeting established goals or they are ceasing breastfeeding before desired or recommended. ${ }^{1-3}$ For those infants who either did not begin breastfeeding at all or stopped breastfeeding shortly after birth, the estimated risk of mortality and morbidity increased in the first 6 months of life. Reduced duration of breastfeeding is estimated to be responsible for 823,000 deaths a year among children $<5$ years of age. ${ }^{4}$ Although there is information available regarding the importance of breastfeeding, public awareness is suboptimal ${ }^{5}$ and there is little consensus regarding the most effective method to assure that as many infants as possible are breastfed in early life. ${ }^{6}$ Although follow-up visits with pediatricians and obstetric professionals are the standard of care for new mothers, no official processes exist for regularly providing lactation guidance or education. With new health care models moving toward emphasis in primary preventative medicine, improving breastfeeding adherence could be a simple matter of providing access to Internationally BoardCertified Lactation Consultants (IBCLCs). The American College of Obstetricians and Gynecologists specifically includes lactation support in the 2018 report on optimizing postpartum (PP) care. ${ }^{7}$

\section{Background and significance}

The World Health Organization defines exclusive breastfeeding as an infant consuming human milk only (including milk expressed or from a wet nurse), with no other food or drink, not even water. ${ }^{8}$ There is no current standardized phrase to indicate infants who are fed solely through the

\footnotetext{
${ }^{1}$ Department of Health and Kinesiology, College of Nursing and Health Sciences, University of Texas at Tyler, Tyler, Texas.

${ }^{2}$ Department of Emergency Medicine, University of California Davis Medical Center, Sacramento, California.
} 
transfer of milk at the breast, ${ }^{9,10}$ yet the distinction between exclusive breastfeeding (per the WHO definition) and what we have deemed "exclusive direct breastfeeding" (EDB) is an important one, particularly for research on health outcomes and should be well defined by the researcher so that the internal validity of the study is not violated.

Early termination of breastfeeding appears to peak during the first week PP and again between 2 weeks and 2 months. There is a decline in the rate of EDB for the first month of life with the greatest observed decline in the first 2 weeks. ${ }^{2}$ A review of interventions to improve breastfeeding duration found that the outcomes of interventions were inconsistent. ${ }^{11}$ In a subsequent update of that review, the authors reported that the most successful interventions were relatively lengthy and were implemented by way of telephone contacts, text message, or through a website. ${ }^{12,13}$ Current breastfeeding interventions are scattered, ${ }^{14}$ without a clear sustainable system for improvement, and although interventions appear to impact selected outcomes, no significant implications are currently acknowledged for clinical practice. ${ }^{15}$ It has been posited that ideal interventions aiming to support breastfeeding should involve primary care physicians, ${ }^{16}$ which may be impractical for already overworked physicians. Although no specific strategy has been identified, it is suspected that breastfeeding support is likely to be more effective in settings with higher breastfeeding initiation rates. ${ }^{12}$ Lower initiation rates are evident for those women who have lower socioeconomic status and less education, ${ }^{17}$ which is associated with limited awareness of resources and information, and little or no access to lactation experts.

Given the lack of definitive success for interventions to increase breastfeeding duration, more effective methods need to be introduced and evaluated to decrease the troubling disparity that exists between initiating breastfeeding and meeting recommended breastfeeding goals.

We describe in this study the evaluation of a cost-effective easy-to-deliver strategy provided to an at-risk population attending a primary care clinic in the normal course of postnatal care. The objective of this strategy was to deliver standard-of-care feeding assessments performed by an IBCLC to all mother-infant dyads attending clinic, improving the standard of care for all, independent of their stated feeding method. The strategy was intended to affect informed decision-making and increase adherence to breastfeeding recommendations. In this study, we were interested to evaluate EDB, as it is associated with sustained durations of breastfeeding. ${ }^{18}$

\section{Methods}

\section{Study design and data source}

A retrospective comparison birth cohort study was conducted with 640 mother-infant dyads utilizing usual charting. Although no intentional interventions were conducted, the researcher had received grant funding by the hospital system to provide lactation consulting in the primary care clinic for a specified period. At the end of that period, the medical records were deidentified and provided to the researcher for analysis to compare breastfeeding rates between those dyads who received the feeding assessments and those who did not. The primary objective of the chart analysis was to determine whether there was any correlation to breastfeeding outcomes and the provision of professional lactation care delivered to mother-infant dyads by an IBCLC in the first 2 weeks PP. The study was approved by the researcher's academic institution and the hospital institutional review boards.

The setting was a hospital-affiliated primary care clinic that served the underinsured and uninsured in the community. Patients attending clinic received the standard prenatal health package that included two well-baby care visits in the first 2 weeks of life and maternal PP care at 6 weeks PP, for a total of three clinic visits. An examination room separate from that used by the physicians was allocated to the IBCLC conducting the feeding assessments.

The sample included all mother-infant dyads attending the clinic for a 2-month period of 40 clinic days. Every motherinfant dyad received standard-of-care feeding assessment provided by an IBCLC, including those mother-infant dyads not breastfeeding and exclusively formula feeding.

\section{Procedures}

Standard of care was provided to every mother-infant dyad equally regardless of ethnicity, socioeconomic status, gender identity, or weight. This consisted of a feeding assessment and an interview with the IBCLC, which included evaluation of the infant's oral cavity, physical breast assessment, and observation of infant-mother feeding behaviors. The initial assessment interview included questions regarding the maternal feeding method intended during pregnancy and being followed at the time of that visit, whether the dyad had received assistance from an IBCLC while in the hospital, Women Infants and Children (WIC) status/eligibility, and if there was prior experience with breastfeeding an infant. Breast care education was provided to all dyads at day 3 and included hand expressing techniques as well as comfort measures for minimizing engorgement (including the use of the infant to remove milk for maternal breast comfort). Information on recognizing infant feeding cues was provided to all dyads at each visit. Standard of care on day 14 included evaluation of the infant's oral cavity, physical breast assessment, and observation of infant-mother feeding behaviors, and addressing any feeding concerns. Typically, the dyad was seen by the IBCLC after the physician had completed the well-infant check and charting was completed by the lactation consultant during normal duties.

At the end of the specified project period, data were deidentified by designated clinic staff. The de-identified data collected from the medical records included the following: demographics, dates of clinic visits, intended feeding method (prenatal and at each visit), participation in the WIC supplemental food program, whether they received help in the hospital with breastfeeding from an IBCLC, and if they had any experience with breastfeeding. The primary outcome of interest was the prevalence of breastfeeding at 6 weeks PP categorized into two outcomes: EDB and any breastfeeding (AB). For this study, the outcome variable "exclusive direct breastfeeding (EDB)" was defined as giving maternal milk directly from the breast as the only food source, with no other foods or liquids, other than vitamins or medications, being given to the infant. The outcome variable "any breastfeeding (AB)" contained participants who identified as feeding infants directly at the breast "sometimes" or "frequently" in 
addition to other feeding methods, such as expressed milk and infant formula with a bottle.

\section{Statistical analysis}

The demographic data were summarized using descriptive statistics. Multiple logistic regression was used to assess the two models. The first model had EDB at 6 weeks as the outcome of interest. The second model had AB at 6 weeks as the outcome of interest. Odds ratios were calculated for these independent variables to determine the probability of exclusive breastfeeding at 6 weeks PP. Variables that were not statistically significant were removed from the final model.

\section{Results}

A total of 640 mother-infant dyads were enrolled as shown in Figure 1. Data from all three visits were obtained for 491 mother-infant dyads. Based on responses in chart history, $61.0 \%$ stated they had received help from an IBCLC in the hospital and $40.9 \%$ had previous experience with breastfeeding. The feeding method intention stated prenatally was recorded at the initial interview: $47.9 \%(N=307)$ of the mothers intended to exclusively breastfeed for at least 6 weeks, with $42.0 \%(N=269)$ planning to use both human milk and formula, and $10.0 \%(N=64)$ intended to use only formula. At the day 3, day 14, and at 6 weeks PP the actual feeding method is given in Table 1.

There was no difference in mean age of the dyads at each visit. The mean age of the mothers was 24.5 years of age (range 16-34 years of age). The mean age of the infants at the first visit was 3.4 days. The mean age of the infants at the second visit was 14.8 days as seen in Table 2 .

Of the mother-infant dyads, at the day 3 visit, there were $371(57.9 \%)$ male and $269(42.1 \%)$ female infants. Of the dyads with complete data $(N=491)$ at 6 weeks PP, there were $295(60.1 \%)$ male and $196(39.9 \%)$ female infants.

Based on the total enrolled $(N=640)$, the ethnicity of the mothers was reported as $40.9 \%$ Hispanic, $29.0 \%$ Caucasian, $20.0 \%$ black, $4.1 \%$ Asian, $5.0 \%$ Native American, and $1.3 \%$ other. Of the total enrolled, 149 participants did not attend the 6-week appointment; 58 (38.9\%) were Hispanic, 4 (28.9\%) were Caucasian, 33 (22.1\%) were black, 5 (3.4\%) were Asian,

\section{Ethnicity $\mathrm{N}=640$}

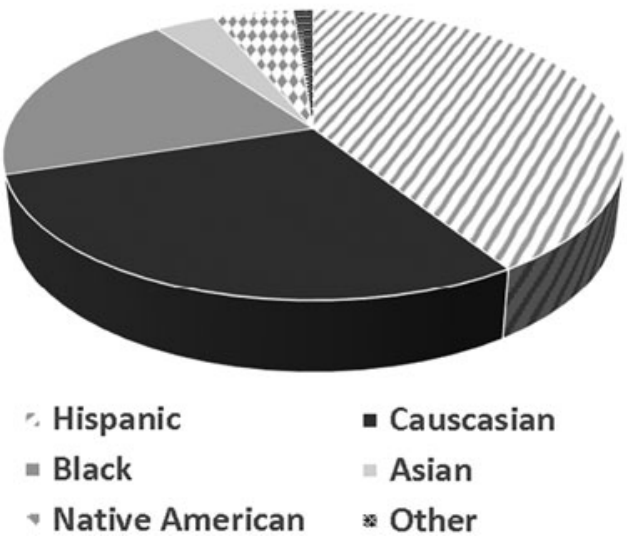

FIG. 1. Participant ethnicity at initial interview.
Table 1. Intention and Actual Feeding Method AT EACH VISIT

\begin{tabular}{lcccc}
\hline & Intention & Day 3 & Day 14 & 6 Weeks \\
\hline EDB, $n(\%)$ & $307(48)$ & $256(40)$ & $278(53)$ & $295(60)$ \\
AB, $n(\%)$ & $269(42)$ & $342(53)$ & $212(40)$ & $157(32)$ \\
FF, $n(\%)$ & $64(10)$ & $42(7)$ & $39(7)$ & $39(8)$ \\
Total & 640 & 640 & 529 & 491 \\
\hline
\end{tabular}

$\mathrm{AB}$, any breastfeeding; EDB, exclusive direct breastfeeding; FF, formula only feeding.

$8(5.4 \%)$ were Native American, and $2(1.3 \%)$ were other. There was no significant difference by participants' ethnicity between those at initial interview on day 3 data and complete data (shown in Fig. 2). Feeding method by ethnicity at 6 weeks $\mathrm{PP}$ is shown in Table 3.

The models included and controlled for lactation visits (yes/no), intended feeding method (EDB, any use of human milk either directly from the breast or via bottle using either expressed human milk or formula $[\mathrm{AB}]$, and formula only feeding), infant gender (male/female), WIC status (receive WIC, eligible for WIC, unknown, and not WIC eligible), previous experience with breastfeeding (yes/no), and help in the hospital by a lactation consultant (yes/no). The variables not statistically significant and removed by backward elimination were infant gender, WIC status, and help in the hospital by a lactation consultant. The variable categories are listed in Table 4. The AB category consisted of women who provided their infants with milk directly from the breast over a spectrum from sometimes to frequently, in addition to bottle feeding with expressed milk and infant formula and would not be classified as exclusively breastfeeding by the WHO definition as they did receive foods (infant formula) other than human milk.

For Model 1 (Table 5), the variables infant gender, WIC status, help in the hospital by a lactation consultant, and prior breastfeeding experience were not significant and were removed from the model. The visits and feeding method were significant. For the visits, there was no significant difference in EDB rates between maternal/infant dyads who were not seen by an IBCLC in the early weeks versus mother-infant dyad who had an IBCLC visit at 2 weeks PP. There was a significant difference in exclusive breastfeeding rates between mother-infant dyads who had IBCLC visits at 2 weeks PP and those who had an IBCLC in the first week PP, typically on day $3 \mathrm{PP}$, with those seen at day 3 having 2.6 times increased odds ratio of EDB at 6 weeks $(p \leq 0.0111)$. The dyads seen both at 3 days and 2 weeks PP had 3.3 times increased odds of exclusively breastfeeding at 6 weeks $(p \leq 0.0001)$. For the intended feeding variable, there was a statistically significant difference in EDB rates between

Table 2. Mean Age at Visit

\begin{tabular}{lc}
\hline & Mean age $(S D)$ \\
\hline Mother & 24.5 years $( \pm 1.3)$ \\
Infant-week 1 visit & 3.4 days $( \pm 0.1)$ \\
Infant-week 2 visit & 14.8 days $( \pm 0.38)$ \\
Infant-week 6 visit & 43.9 days $( \pm 0.85)$ \\
\hline
\end{tabular}

$\mathrm{SD}$, standard deviation. 


\section{Ethnicity $\mathrm{N}=491$}

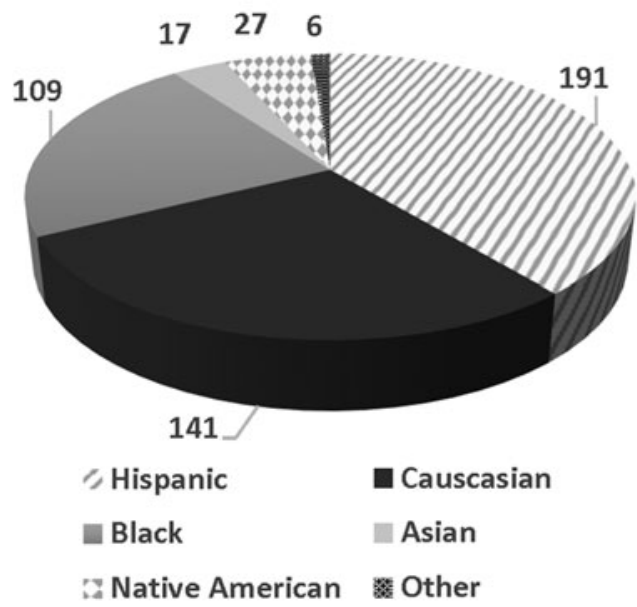

FIG. 2. Participant ethnicity at 6 weeks postpartum.

mother-infant dyads who wanted to exclusively breastfeed before infant birth and mother-infant dyads who intended to use mixed feeding before birth.

In Model 2 (Table 6), the variables gender, WIC status, help in the hospital by a lactation consultant, and prior breastfeeding experience were not significant and were removed from the model by backward elimination with $\mathrm{AB}$ at the 6 weeks visit as the outcome of interest. There was no statistically significant difference in the rate of $A B$ between mother-infant dyads who were seen only at 2 weeks PP and mother-infant dyads not seen by an IBCLC during the first 2 weeks PP. Visits and intended feeding method were significant. For mother-infant dyads who were seen during the first week PP, there was a significant difference in the rates of $A B$ between mother-infant dyads seen during the first week and at 2 weeks versus mother-infant dyad who were seen only at 2 weeks PP. These mother-infant dyads had 2.6 times increased odds of AB at 6 weeks PP compared with motherinfant dyads only seen at 2 weeks PP. There was a difference for the variable of intended feeding in the rates of $A B$ between dyads who intended to exclusively breastfeed before birth and dyads who intended to use a mixed feeding method before birth. These mother-infant dyads had 3.5 times decreased odds of AB compared with mother-infant dyads who wanted to exclusively breastfeed $(p>0.0001)$. Although intention to breastfeed in this study appears to be an important predictor of breastfeeding, the use of any bottles decreased the odds ratio of $\mathrm{AB}$ by 3.4 times at 6 weeks PP.

This study has several limitations. The length of the study was short-only a 2-month period. Continuing the study for a longer time could provide different results. Only two cate-

Table 3. Feeding Method by Ethnicity at 6 Weeks Postpartum

Native

Hispanic Caucasian Black Asian American Other

\begin{tabular}{lrrrrrr}
\hline EDB & 115 & 85 & 65 & 10 & 16 & 4 \\
AB & 61 & 45 & 35 & 5 & 9 & 2 \\
FF & 15 & 11 & 9 & 2 & 2 & 0 \\
Total & 191 & 141 & 109 & 17 & 27 & 6 \\
\hline
\end{tabular}

Table 4. Percentages of Breastfeeding

by Variable Category at 6-Week Postpartum for

ExClusive BreastfeEding (EB) AND ANY BREASTFEEDING (AB)

\begin{tabular}{|c|c|c|}
\hline \multirow[b]{2}{*}{ Variable category } & \multicolumn{2}{|c|}{$\begin{array}{l}\text { Feeding at } \\
6 \text { weeks PP }\end{array}$} \\
\hline & EB & $\mathrm{AB}$ \\
\hline \multicolumn{3}{|l|}{ Infant total, \% } \\
\hline Male & 62 & 71 \\
\hline Female & 56 & 68 \\
\hline \multicolumn{3}{|l|}{ WIC status, \% } \\
\hline Receive WIC & 50 & 65 \\
\hline Eligible for WIC & 66 & 72 \\
\hline Unknown WIC eligibility & 76 & 76 \\
\hline Not WIC eligible & 65 & 73 \\
\hline \multicolumn{3}{|c|}{ Previous breastfeeding experience, $\%$} \\
\hline Yes & 60 & 71 \\
\hline No & 59 & 67 \\
\hline \multicolumn{3}{|c|}{ Received IBCCLC help in hospital, \% } \\
\hline Yes & 61 & 71 \\
\hline No & 56 & 65 \\
\hline \multicolumn{3}{|l|}{ Intended feeding method, $\%$} \\
\hline Exclusive breastfeeding & 72 & 82 \\
\hline Formula feeding & 8 & 8 \\
\hline Mixed feeding & 44 & 56 \\
\hline \multicolumn{3}{|l|}{ IBCLC visits, \% } \\
\hline Six weeks only & 40 & 60 \\
\hline First and sixth weeks & 63 & 68 \\
\hline Second and sixth weeks & 43 & 59 \\
\hline First, second, and sixth weeks & 70 & 77 \\
\hline Total, \% & 60 & 69 \\
\hline
\end{tabular}

IBCLC, Internationally Board-Certified Lactation Consultant; PP, postpartum; WIC, Women Infants and Children.

gories were used as outcome variables: $\mathrm{EDB}$ and $\mathrm{AB}$. Grouping the variables separately for use of any human milk and only human milk may have provided valuable information. The participants in the study were uninsured or underinsured. It is unknown if this strategy would benefit a different population. In addition, the IBCLC doing the feeding assessments was highly experienced with an advanced skill set accustomed to managing lactation challenges, which could bias the results.

Table 5. ExClusive Breastfeeding at 6 Weeks Postpartum

\begin{tabular}{llll}
\hline Variable & $\begin{array}{l}\text { Odds } \\
\text { ratio }\end{array} 95 \%$ CI & $\mathrm{p}$ \\
\hline
\end{tabular}

\begin{tabular}{lcccc}
$\begin{array}{l}\text { Intended feeding method } \\
\text { Exclusive }\end{array}$ & & & \\
$\quad$ breastfeeding & Reference & & \\
Formula feeding & 0.029 & $0.004-0.234$ & $<0.001$ \\
Mixed feeding & 0.291 & $0.168-0.503$ & $<0.001$ \\
IBCLC visits & & & \\
Six weeks only & 1.022 & $0.132-7.893<0.001$ \\
Day 3 and 6 weeks & 2.546 & $1.238-5.236<0.011$ \\
Day 14 and 6 weeks & Reference & & \\
Day 3, day 14, and & 3.341 & $1.796-6.215<0.001$ \\
6 weeks & & & \\
\hline
\end{tabular}


Table 6. Any Breastfeeding at 6 Weeks Postpartum

\begin{tabular}{lccc}
\hline Variable & Odds ratio & $95 \%$ CI & $\mathrm{p}$ \\
\hline Intended feeding method & & & \\
$\quad$ Exclusive breastfeeding & Reference & & $<0.003$ \\
$\quad$ Formula feeding & 0.017 & $0.002-0.137$ & $<0.001$ \\
$\quad$ Mixed feeding & 0.282 & $0.159-0.500$ & $<0.002$ \\
IBCLC visits & 1.509 & & $<0.001$ \\
$\quad$ Six weeks only & 1.567 & $0.157-14.555$ & $<0.004$ \\
Day 3 and 6 weeks & Reference & & \\
Day 14 and 6 weeks & 2.628 & $1.357-5.092$ & \\
Day 3, day 14, and 6 weeks & & & \\
\hline
\end{tabular}

\section{Discussion}

Interventions should include early and convenient access to resources. With the expansion of preventative health care, primary prevention through education and regulated inperson assistance is invaluable. Although the actions reported in this study were not an intended intervention, the care received was correlated with higher odds of breastfeeding. This study demonstrated that adding feeding assessment as a standard part of well-baby care was associated with increased odds of breastfeeding continuation at 6 weeks PP in a population with relatively low breastfeeding initiation, and historically short duration of breastfeeding.

Some of the women who intended to use only formula reported some breastfeeding, whereas three dyads in this group were exclusively breastfeeding at 6 weeks PP reportedly due to putting the infant to breast to help relieve initial engorgement during involution. This strongly indicates that providing standard-of-care feeding assessment and education in the first week PP may lead to an increase in breastfeeding duration. By 2 weeks PP any challenges with breastfeeding have occurred and compounded, making them difficult to resolve. The women in this study did not have ready access to either a manual or electric breast pump in the first 2 weeks of lactation. Teaching them hand expression may have been a factor in breastfeeding continuation. ${ }^{19}$

In this predominantly Hispanic group (40.9\%), it was not surprising that $41.9 \%$ of the total participants intended to feed their infants both from the breast and the bottle as this is reported to be a common practice among Hispanic women. ${ }^{20}$ However, ethnicity was not significant for either feeding outcome at 6 weeks PP.

It has been suggested that interventions aiming to support breastfeeding should involve primary care physicians as a component of routine health care and preventive advocacy. ${ }^{14}$ Formula ads and coupons are readily available in many physicians' offices, but it can be difficult to find comparative accommodations for breastfeeding support. Medical training is largely unable to incorporate many opportunities to learn breastfeeding techniques and recommendations. ${ }^{21}$ However, additional education for pediatricians and working with lactation consultants could be used to improve access to lactation assessment and management. ${ }^{22}$

Although primary care practitioners are the main providers for organizing access to resources for many patients, unfortunately, in a lactation role, many practitioners are limited in experience and education on the subject. Lactation management is not a part of regular physician training and most residents lack the knowledge and training to manage breastfeeding difficulties, ${ }^{23}$ and current medical resident training is being implemented in a limited way. ${ }^{24}$

Providing community-based care is also problematic. It has been reported that early home-based and communitybased support was difficult to implement. ${ }^{25}$ As telemedicine is on the rise, it has found an application in this field as well. In a study on rural women the authors concluded that "telelactation" was useful to provide lactation support for those who did not have local, readily available access to qualified lactation resources. ${ }^{26}$ Although virtual consultations may play a role in improving postdelivery contact and increasing exclusive breastfeeding rates in communities with resource deficits, ${ }^{27,28}$ we believe that these do not and should not replace access to in-person care.

The cost and time required for intensive interventions may be impractical for many communities, but standard lactation visits as part of an integrated preventive health system would be a fiscally responsible approach. As opposed to implementing a new system, supplementing the current primary care provider model with an IBCLC as part of the health care team, as in the strategy reported in this study, was an efficient easy means to establish convenient care for patients.

\section{Conclusions}

The neonatal period is a critical time to provide support for the breastfeeding dyad. The data reported in this study support the practice of outpatient lactation management beginning the first few days of lactation as a possible means of increasing the duration of exclusive breastfeeding. This study establishes the efficacy of breastfeeding support with reliable and convenient access to resources and assistance by an IBCLC. We believe that an IBCLC should be an integral part of primary care groups. We posit that early lactation assessment as a part of community clinics could fill the "support" gap that new families need to meet their breastfeeding goals. Early evaluation with IBCLCs is an essential aspect of breastfeeding success.

Additional support is needed by women in the early PP period to increase the number of mother-infant dyads achieving the recommendations for breastfeeding outcomes. We agree with Riddle and Nommsen-Rivers that, "Breastfeeding mother-infant dyads should be followed closely until lactation is well established." 29 As a retrospective chart analyses we are able to identify a strong correlation between improved access to lactation professionals and adherence to active breastfeeding recommendations, and although it is 
often well accepted that preventative health care leads to improved outcomes it is difficult to establish a causative relationship due to the extensive compounding variables. Cultural practices and individual compliance play a large role in the actuation of these principles. Additional research is needed to determine any long-term effects of early IBCLC feeding assessment on the duration of breastfeeding and could be helpful to replicate the results identified in this study.

\section{Author Disclosure Statement}

The authors have no conflict of interest.

\section{References}

1. Nelson JM, Li R, Perrine CG, Scanlon KS. Changes in mothers' intended duration of breastfeeding from the prenatal to neonatal periods. Birth 2018;45:178-183.

2. Patil CL, Turab A, Ambikapathi R, et al. MAL-ED network. Early interruption of exclusive breastfeeding: Results from the eight-country MAL-ED study. J Health Popul Nutr 2015;34:10.

3. Sriraman NK, Kellams A. Breastfeeding: What are the barriers? Why women struggle to achieve their goals. J Women's Health (Larchmt) 2016;25:714-722.

4. Victora CG, Bahl R, Barros AJ, et al.; Lancet Breastfeeding Series Group. Breastfeeding in the 21st century: Epidemiology, mechanisms, and lifelong effect. Lancet 2016; 387:475-490.

5. Nelson JM, Li R, Perrine CG, et al. Public opinions about infant feeding in the United States. Birth 2016;43:313-319.

6. Haroon S, Das JK, Salam RA, et al. Breastfeeding promotion interventions and breastfeeding practices: A systematic review. BMC Public Health 2013;13:S20.

7. ACOG Committee Opinion No. 736: Optimizing postpartum care. Obstet Gynecol 2018;131:e140-e150.

8. World Health Organization. Infant feeding recommendation. Definition of exclusive breastfeeding. Available at: www .who.int/nutrition/topics/infantfeeding_recommendation/en Accessed February 21, 2019.

9. Noel-Weiss J, Boersma S, Kujawa-Myles S. Questioning current definitions for breastfeeding research. Int Breastfeed J 2012;7:9.

10. Still R, Marais D, Hollis JL. Mothers' understanding of the term "exclusive breastfeeding": A systematic review. Matern Child Nutr 2017;13:1-20.

11. Beake S, Bick D, Narracott C, Chang YS. Interventions for women who have a caesarean birth to increase uptake and duration of breastfeeding: A systematic review. Matern Child Nutr 2017;13:1-13.

12. Skouteris H, Nagle C, Fowler M, Kent B, Sahota P, Morris $\mathrm{H}$. Interventions designed to promote exclusive breastfeeding in high-income countries: A systematic review. Breastfeed Med 2014;9:113-127.

13. Skouteris H, Bailey C, Nagle C, Hauck Y, Bruce L, Morris $\mathrm{H}$. Interventions designed to promote exclusive breastfeeding in high-income countries: A systematic review update. Breastfeed Med 2017;12:604-614.

14. Galipeau R, Baillot A, Trottier A, et al. Effectiveness of interventions on breastfeeding self-efficacy and perceived insufficient milk supply: A systematic review and metaanalysis. Matern Child Nutr 2018;14:e12607.

15. Johnson A, Kirk R, Rosenblum KL, Muzik M. Enhancing breastfeeding rates among African American women: A systematic review of current psychosocial interventions. Breastfeed Med 2015;10:45-62.

16. Labarere J, Gelbert-Baudino N, Ayral AS, et al. Efficacy of breastfeeding support provided by trained clinicians during an early, routine, preventive visit: A prospective, randomized, open trial of 226 mother-infant pairs. Pediatrics 2005; 115:E139-E146.

17. Thomson JL, Tussing-Humphreys LM, Goodman MH, Landry AS, Olender SE. Low rate of initiation and short duration of breastfeeding in a maternal and infant home visiting project targeting rural, Southern, African American women. Int Breastfeed J 2016;12:15.

18. Pang WW, Bernard JY, Thavamani G, et al. Direct vs. expressed breast milk feeding: relation to duration of breastfeeding. Nutrients 2017;9:pii:E547.

19. Flaherman VJ, Gay B, Scott C, Avins A, Lee KA, Newman TB. Randomized trial comparing hand expression with breast pumping for mothers of term newborns feeding poorly. Arch Dis Child Fetal Neonatal Ed 2012;97:F18-23.

20. Bartick M, Reyes C. Las dos cosas: An analysis of attitudes of Latina women on non-exclusive breastfeeding. Breastfeed Med 2012;7:19-24.

21. Esselmont E, Moreau K, Aglipay M, Pound CM. Residents' breastfeeding knowledge, comfort, practices, and perceptions: Results of the Breastfeeding Resident Education Study (BRESt). BMC Pediatr 2018;18:170.

22. Sriraman NK. Promote breastfeeding in the outpatient setting: It's easy! Curr Probl Pediatr Adolesc Health Care 2017;47:311-317.

23. Albert JB, Heinrichs-Breen J, Belmonte FW. Development and evaluation of a lactation rotation for a pediatric residency program. J Hum Lact 2017;33:748-756.

24. Taylor JS, Bell E. Medical education and leadership in breastfeeding medicine. Breastfeed Med 2017;12:476-478.

25. McLachlan HL, Forster DA, Amir LH, et al. Supporting breastfeeding in local communities (SILC) in Victoria, Australia: A cluster randomised controlled trial. BMJ Open 2016;6:e008292.

26. Demirci J, Kotzias V, Bogen DL, Ray KN, Uscher-Pines L. Telelactation via mobile app: Perspectives of rural mothers, their care providers, and lactation consultants. Telemed J E Health 2018;13. [Epub ahead of print]; DOI: 10.1089/ tmj.2018.0113.

27. Harari N, Rosenthal MS, Bozzi V, et al. Feasibility and acceptability of a text message intervention used as an adjunct tool by WIC breastfeeding peer counsellors: The LATCH pilot. Matern Child Nutr 2018;14:1-11.

28. Xie RH, Tan H, Taljaard M, et al. The impact of a maternal education program through text messaging in Rural China: Cluster randomized controlled trial. JMIR Mhealth Uhealth 2018;6:e11213.

29. Riddle SW, Nommsen-Rivers LA. Low milk supply and the pediatrician. Curr Opin Pediatr 2017;29:249-256.

Address correspondence to: Jimi Francis, PhD, IBCLC, RDN Department of Health and Kinesiology College of Nursing and Health Sciences University of Texas at Tyler 3900 University Boulevard Tyler, TX 75799

E-mail: jfrancis@uttyler.edu 